\title{
ON THE EXISTENCE OF POINT COUNTABLE BASES IN MOORE SPACES
}

\author{
G. M. REED
}

\begin{abstract}
In this paper, the author answers in the negative two questions raised by E. E. Grace and R. W. Heath concerning the existence of point countable bases in Moore spaces. These answers are obtained by a general construction technique developed by the author which associates to each first countable $T_{2}$-space a Moore space.
\end{abstract}

In [4], F. B. Jones showed that if $2^{\boldsymbol{N}_{0}}<2^{\boldsymbol{N}_{1}}$, then every separable normal Moore space is metrizable. Hence, since each separable Moore space with a point countable base is known to be metrizable ([8] and [6]), the following question has often been raised: If $2^{\boldsymbol{N}_{0}}<2^{\boldsymbol{N}_{1}}$, must each normal Moore space have a point countable base? In [1], E. E. Grace and R. W. Heath considered metrizability of metacompact (pointwise paracompact) Moore spaces and of Moore spaces with a point countable base. Also in [1], they raised the following two questions whose solutions would be beneficial to a solution of the above question: (1) A Moore space $S$ is said to have property $P$ provided that for each separable subset $M$ of $S$ and each open covering $H$ of $S$, there exists an open covering $K$ of $S$ such that $K$ refines $H$, and $M$ intersects only countably many members of $K$. Grace and Heath noted that if $2 \boldsymbol{N}_{0}<2^{\boldsymbol{N}_{1}}$, then each normal Moore space has property $\mathrm{P}$.

Question 1 Does each Moore space with property $\mathrm{P}$ have a point countable base?

(2) Question 2. Is each Moore space with a point countable base metacompact?

In [7], the author described a technique which associates to each first countable $T_{2}$-space $X_{0}$ a Moore space $X$. In this paper it is shown that (i) if $X_{0}$ is the space of countable ordinals with the order topology, then the associated space $X$ provides a negative answer to Question 1 , and (ii) if $X_{0}$ is a certain space given in [2], then the associated space $X$ provides a nega-

Received by the editors May 4, 1973.

AMS (MOS) subject classifications (1970). Primary 54E30, 54G20; Secondary $54 \mathrm{D} 20$. 
tive answer to Question 2. Note that a negative answer to Question 2 was announced by Heath in [3], although to the author's knowledge such an example has never appeared in print. However, Professor Heath has shown his example to the author, and the author gratefully acknowledges that his solution to Question 2 was obtained with hindsight of Heath's example.

A development for a space $S$ is a sequence $G_{1}, G_{2}, \ldots$ of open coverings of $S$ such that (1) $G_{i+1} \subset G_{i}$ for each $i$, and (2) if $p \in S$ and $D$ is an open set containing $p$ then there exists an $n$ such that each element of $G_{n}$ containing $p$ is contained in $D$. A Moore space is a regular $T_{1}$-space which has a development. The statement that a collection $H$ of point sets is point finite (point countable) means that no point belongs to infinitely (uncountably) many elements of $H$. A space $S$ is metacompact provided for each open covering $G$ of $S$ there exists a point finite open covering $H$ of $S$ which refines $G$ and covers $S$. It follows immediately that each metacompact Moore space has a point countable base.

Theorem 1. There exists a Moore space $X$ such that for each open covering $H$ of $X$ and each separable subset $M$ of $X$ there exists an open cov. ering $K$ of $X$ such that $K$ refines $H$, and $M$ intersects only countably many members of $K$ but $X$ does not have a point countable base.

Proof. Denote by $X_{0}$ the space of countable ordinals with the order topology. For each $x \in X_{0}$ such that $x$ is a limit ordinal, denote by $x_{1}, x_{2}$, ... a sequence of elements of $X_{0}$ which converges to $x$. Then, for each $i$, let $u_{i}(x)=\left\{y \in X_{0} \mid x_{i}\right.$ precedes $y$ and either $y$ is $x$ or $y$ precedes $\left.x\right\}$. For each $x \in X_{0}$ such that $x$ is not a limit ordinal in $X_{0}$ and for each $i$, let $u_{i}(x)=\{x\}$. Note that for each $x \in X_{0}, u_{1}(x), u_{2}(x), \ldots$ forms a local base for $x$ in $X_{0}$. Now, denote by $S_{1}$ a copy of $X_{0}$ and for each positive integer $i$, denote by $S_{(1, i)}$ a unique copy of $X_{0}$ distinct from $S_{1}$. Let $X=S_{1} \cup$ $\left(\bigcup_{i=1}^{\infty} S_{(1, i)}\right)$ and for each $p \in X$, denote by $x_{p}$ the element of $X_{0}$ which is identified with $p$. If $p \in X$ and $j$ is a positive integer, define $g_{j}(p)$ as follows: (1) If $p \in S_{(1, i)}$ for some $i$, let $g_{j}(p)=\{p\}$. (2) If $p \in S_{1}$, let $g_{j}(p)=$ $\{p\} \cup\left\{q \in S_{(1, i)} \mid i \geq j\right.$ and $x_{q} \in u_{i}\left(x_{p}\right)$ in $\left.X_{0}\right\}$. It follows from [7] that if $G_{i}=$ $\left\{g_{j}(p) \mid p \in X\right.$ and $\left.j \geq i\right\}$ for each $i$, then $G_{1}, G_{2}, \ldots$ is a development for the nonnormal Moore space $X$. To see that $X$ is not normal consider $H=$ $\left\{p \in S_{1} \mid x_{p}\right.$ is a limit ordinal in $\left.X_{0}\right\}$ and $K=\left\{p \in S_{1} \mid x_{p}\right.$ is not a limit ordinal in $\left.X_{0}\right\}$. It is easily seen that $H$ and $K$ are two mutually exclusive closed sets in $X$ which cannot be separated by mutually, exclusive open sets.

Claim 1. $X$ is locally separable. To see this, for each $p \in X$ and each 
$j$, consider $g_{j}(p)$. Note that since each initial segment in $X_{0}$ is countable, by definition so is $g_{j}(p)$.

Claim 2. Each separable subset $M$ of $X$ is countable. For if $M$ is separable, $M \cap S_{(1, i)}$ is countable for each $i$, since each point of $X$ not on $S_{1}$ is isolated. Thus $s, T=M \cap\left(\bigcup_{i=1}^{\infty} S_{(1, i)}\right)$ is countable. Now, let $t \in S_{1}$ such that if $p \in T$ then $x_{p}$ is $x_{t}$ or $x_{p}$ precedes $x_{t}$ in $X_{0}$. It follows that if $q \in S_{1}$ and $x_{q}$ follows $x_{t}$ in $X_{0}$, then $q$ is not in the closure of $T$ in $X$. Thus, since no point of $S_{1}$ is a limit point of $S_{1}$, there are at most countably many points $q$ of $S_{1} \cap M$ such that $x_{q}$ follows $x_{t}$ in $X_{0}$. Hence, $M$ is countable.

Claim 3. If $M$ is a separable subset of $X$ and $H$ is an open covering of $X$, then there exists an open covering $K$ of $X$ such that $K$ refines $H$, and $M$ intersects only countably many elements of $K$. By Claim 2, $M$ is countable. Thus, let $t \in S_{1}$ such that if $p \in M$ then either $x_{p}$ is $x_{t}$ or $x_{p}$ precedes $x_{t}$ in $X_{0}$. Now, if $q \in X$ such that $x_{q}$ does not follow $x_{t}$ in $X_{0}$, let $g(q)$ be an open set containing $q$ which is contained in an element of $H$. If $q \in X$ such that $x_{q}$ follows $x_{t}$, let $g(q)$ be an open set containing $q$ which is contained in an element of $H$ and which does not intersect $\left\{p \in X \mid x_{p}\right.$ precedes $x_{t}$ or $x_{p}$ is $\left.x_{t}\right\}$. If $K=\{g(q) \mid q \in X\}$, then $K$ has the desired properties.

Claim 4. $X$ does not have a point countable base. By the proof of $[8$, Theorem 1], each locally separable Moore space with a point countable base is metrizable. This completes the proof.

Theorem 2. There exists a Moore space with a point countable base that is not metacompact.

Proof. Denote by $X_{0}$ a well-ordered uncountable subset of the $x$-axis such that each initial segment is countable. Let $T_{1}$ denote the usual topology on $X_{0}$ and let $T_{2}$ denote the collection of final segments of $X_{0}$. Now, let $T$ denote the supremum of $T_{1}$ and $T_{2}$. The space $\left(X_{0}, T\right)$ was given in [2] as an example of a hereditarily Lindelöf, first countable $T_{2}$-space which is not separable. For each point $x \in X_{0}$ and each $i$, let $u_{i}(x)=\left\{y \in X_{0} \mid y \in\right.$ $(x-1 / i, x+1 / i)$ in $T_{1}$ and either $x$ is $y$ or $x$ precedes $y$ in the wellordering of $\left.X_{0}\right\}$. Then for each $x \in X_{0}, u_{1}(x), u_{2}(x), \ldots$ is a local base for $x$ in $\left(X_{0}, T\right)$. As in the proof of Theorem 1 , denote by $S_{1}$ a copy of $X_{0}$ and for each positive integer $i$, denote by $S_{(1, i)}$ a unique copy of $X_{0}$ distinct from $S_{1}$. Let $X=S_{1} \cup\left(\bigcup_{i=1}^{\infty} S_{(1, i)}\right)$ and for each $p \in X$, denote by $x_{p}$ the element of $X_{0}$ which is identified with $p$. If $p \in X$ and $j$ is a positive integer, define

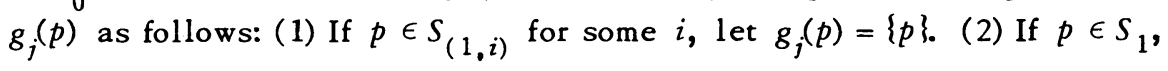


let $g_{j}(p)=\{p\} \cup\left\{q \in S_{(1, i)} \mid i \geq j\right.$ and $x_{q} \in u_{i}\left(x_{p}\right)$ in $\left.\left(X_{0}, T\right)\right\}$. Again, it follows that if $G_{i}=\left\{g_{j}(p) \mid p \in X\right.$ and $\left.j \geq i\right\}$ for each $i$, then $G_{1}, G_{2}, \ldots$ is a development for the Moore space $X$.

Claim 1. $X$ has a point countable base. Observe that for each $p \in X$ and each $i$, if $p \in g_{i}(q)$ for some $q \in X$, then $x_{q}$ is $x_{p}$ or $x_{q}$ precedes $x_{p}$ in the well-ordering of $X_{0}$. Hence, each point $p$ of $X$ is contained in at most countably many elements of $G_{1}$.

Claim 2. $X$ is not metacompact. For suppose that $H$ is a point finite open covering of $X$ which refines $G_{1}$. Note that each element of $H$ contains at most one point of $S_{1}$, and since $S_{1}$ is uncountable, there exists a positive integer $n$ such that $K=\left\{p \in S_{1} \mid g_{n}(p)\right.$ is contained in an el ement of $\left.H\right\}$ is uncountable. Consider $M=\left\{x_{q} \in X_{0} \mid q \in K\right\}$. Since $M$ is uncountable, by [5, Chapter 1, Theorem 6], there exists a point $x \in M$ such that $x$ is a limit point with respect to $T_{1}$ of $\left\{x_{q} \in M \mid x_{q}\right.$ precedes $x$ in the well-ordering of $\left.X_{0}\right\}$. But, it follows that there exists a point $r \in S_{(1, i)}$ such that $x_{r}=x$ and $r$ is contained in infinitely many elements of $H$. This completes the proof.

\section{REFERENCES}

1. E. E. Grace and R. W. Heath, Separability and metrizability in pointwise paracompact Moore spaces, Duke Math. J. 31 (1964), 603-610. MR 29 \#6464.

2. R. S. Countryman, Spaces having a o-monotone base (to appear).

3. R. W. Heath, $A$ non-pointwise paracompact Moore space with a point computable base, Notices Amer. Math. Soc. 10 (1963), 649-650. Abstract \#605-22.

4. F. B. Jones, Concerning normal and completely normal spaces, Bull. Amer. Math. Soc. 43 (1937), 671-677.

5. R. L. Moore, Foundations of point set theory, rev. ed., Amer. Math. Soc. Colloq. Publ., vol. 13, Amer. Math. Soc., Providence, R. I., 1962.

6. G. M. Reed, On screenability and metrizability of Moore spaces, Canad. J. Math. 23 ( 1971 ), 1087-1092. MR 45 \#1114.

7. - On chain conditions in Moore spaces, General Topology and Appl. (to appear).

8. D. R.Traylor, Concerning metrizability of pointwise paracompact Moore spaces, Canad. J. Math. 16 (1964), 407-411. MR 29 \#1622.

DEPARTMENT OF MATHEMATICS, OHIO UNIVERSITY, ATHENS, OHIO 45701 